H I G H L I G H T S

PLANT GENOMICS

\section{Rice grains bear fruit}

If you measure a genome by the practical benefits of its sequencing, then they come no more important than that of rice (Oryza sativa) the staple crop of over half the population. Moreover, it's a good model for other grasses, including the other global food crops wheat and maize.

Two draft sequences of rice are now reported in Science, both generated by whole-genome shotgun approaches. Jin $\mathrm{Yu}$ and colleagues, at the Beijing Genomics Institute (BGI), have sequenced the subspecies $O$. s. indica, which is commonly cultivated in China and South-east Asia, whereas Stephen Goff and colleagues at Syngenta have sequenced the O. s. japonica subspecies. Controversially, the BGI sequence is available in GenBank, whereas Syngenta's can only be accessed through an agreement similar to that used by Celera for its sequence of the human genome.

BGI have sequenced $362 \mathrm{Mb}$ of the $466 \mathrm{Mb}$ indica genome to fourfold coverage, $\sim 90 \%$ at over $\mathbf{9 9 . 9 \%}$ accuracy. The initial annotation turned up 53,398 complete genes, although this could rise to 64,500 if many partial predictions are confirmed. The japonica genome is slightly shorter at $420 \mathrm{Mb}, 390 \mathrm{Mb}$ of which the Syngenta team sequenced at over sixfold coverage, at $99.8 \%$ accuracy. Their conservative annotation identified 32,277 genes, although the total number might be over 60,000 .

Where the BGI group was able to align their draft to portions of the japonica genome sequenced by the International Rice Genome Sequencing Project, sequence identity was close to $100 \%$. The $14 \%$ that did not match bears the hallmarks of transposon activity evidence of rapid changes of genome size in the grasses.

Comparisons were also made with the Arabidopsis thaliana genome. Eighty-five per cent of

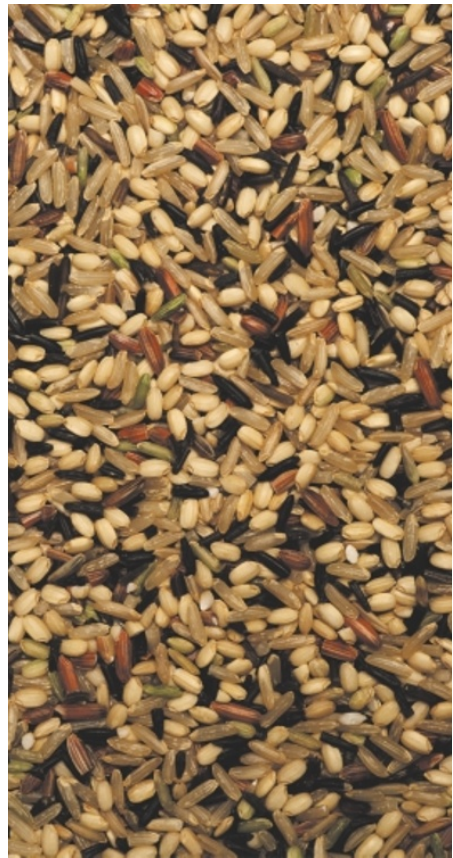

Arabidopsis genes have homologues in rice, although only $\sim 50 \%$ of rice genes have homologues in Arabidopsis, hinting at a major duplication event in rice's evolutionary history. Around onethird of the homologous genes are plant specific. Also, $30 \%$ of the most highly conserved genes between the two plants have no confirmed function, showing that many crucially important plant genes remain to be characterized.

Deeper questions about the architecture and evolution of the rice genome await the finished sequence. Such 'gold standard' approaches are also required if the rice genome is to be used as a model for other grass staples, such as wheat, the large genome and hexaploid status of which make sequencing it impractical.

Christopher Surridge, Senior Editor, Nature

69) References and links ORIGINAL RESEARCH PAPERS Yu, J. et al. A draft sequence of the rice genome (Oryza sativa L. ssp indica). Science 296, 80-92 (2002) | Goff, S. A. et al. A draft sequence of the rice genome (Oryza sativa L. ssp japonica). Science 296, 92-100 (2002)

\section{IN BRIEF}

\section{DEVELOPMENTAL BIOLOGY}

Coordination of chondrogenesis and osteogenesis by fibroblast growth factor 18 .

Liu, Z. et al. Genes Dev. 16, 859-869 (2002)

FGF18 is required for normal cell proliferation and differentiation during osteogenesis and chondrogenesis.

Ohbayashi, N. et al. Genes Dev. 16, 870-879 (2002)

Fibroblast growth factor (FGF) signalling is involved in the development of the vertebrate skeleton. Studies of inherited human skeletal disorders and of mouse knockouts have implicated various FGF receptors (FGFR) in bone and cartilage formation, but until now no ligands have been identified. Now, two papers report that $\mathrm{Fgf1} 18$ is expressed in the regions of bone formation in the mouse embryo, in areas that overlap with Fgfr3 expression. The possibility that Fgf18 is a ligand for Fgfr3 is supported by the similarity of the two knockout phenotypes $\mathrm{Fg}_{\mathrm{f} 18^{-/-}}$embryos have expanded zones of proliferating chondrocytes, and chondrocyte proliferation and differentiation is increased. The delayed ossification seen in Fgf18, but not in Fgfr3 mutants, indicates that Fgf18 probably signals through another FGFR to regulate this process.

\section{HUMAN DISEASE}

Clonally expanded mtDNA point mutations are abundant in individual cells of human tissues.

Nekhaeva, E. et al. Proc. Natl Acad. Sci. USA 99, 5521-5526 (2002)

The interest in somatic mitochondrial DNA (mtDNA) mutations derives from their proposed involvement in ageing and tumour development. The phenotypic expression of a mtDNA variant is thought to occur through its clonal expansion in a cell, until it reaches a frequency of $\sim 90 \%$. Although it has been shown that large mtDNA deletions can be clonally expanded in individual cells, the authors provide the first evidence that the more clinically relevant point mutations can expand in vivo in normal human tissues. By sequencing the mtDNA of individual proliferating and postmitotic cells, they have shown that many cells can accumulate a high proportion of clonally expanded point-mutated mtDNA.

\section{EVOLUTION}

The X chromosome is a hot spot for sexually antagonistic fitness variation.

Gibson, J. R. et al. Proc. R. Soc. Lond. B 269, 499-505 (2002)

The genome is shaped by different evolutionary forces according to whether it resides in a female or male organism. Theory has it that fitness variation for these sexually antagonistic traits - those that are advantageous for one sex but disadvantageous for the other - are enriched on the $\mathrm{X}$ chromosome. Gibson et al. have now provided evidence of this in Drosophila by comparing the fitness variation on 20 cytogenetically cloned X chromosomes to that of the rest of the genome. They show, among other things, that the X chromosome harbours $97 \%$ of the sexually antagonistic fitness variation, but only in adults, when the role of the two sexes diverge. 